\title{
Network Traffic Model for Industrial Environment
}

\author{
Janusz Kolbusz, Stanisław Paszczyński, Senior Member, IEEE, and Bogdan M. Wilamowski, Fellow, IEEE
}

\begin{abstract}
In this paper, a model of LAN traffic is presented. In the model, the most important components influencing the network traffic are taken into account. Namely, the transmission protocols and information buffering, operating systems, and queuing algorithms as well as user behavior in network applications are considered. The model is based on an "on-off" function. The network traffic observed at the physical layer is a superposition of many sequential and self-similar "on-off" processes. It has been shown that the self-similarity of the traffic, measured by the Hurst parameter, changes from almost 1.0 for very low frequencies to 0.5 for high frequencies.
\end{abstract}

Index Terms-Components traffic, network protocols, traffic model.

\section{INTRODUCTION}

C OMPUTER network traffic has a very complex and timedependant structure. In the first model of network traffic, proposed by Erlang in the 1920s, a Poisson process was used in analyzing the traffic in telecommunication networks [3]. These models did not fit the recorded traces, since they did not reflect the packet nature of messages and were not able to capture the fractal behavior of the traffic [10], [13], [22].

Networks with packet switching properties were developed in the 1970s. At the time, most analytical models of computer networks were based on a simplified approach where both intervals between packets and duration of packets were described by exponential relationships. These models were not capable of representing correlations between neighboring events. In these models, the arrival would have a characteristic burst length, which would tend to be smoothed by averaging over a long enough time scale, whereas measurements of real traffic indicate that significant traffic variance burstiness is present on a wide range of time scales.

These deficiencies were partially eliminated in models based on auto regression [4]. Autoregressive models are described by the value of a random variable, or a sequence of random variables, which is defined by the linear combination preceding the random variables. Several such models were already described in [10] and [21]: discrete autoregressive (DAR) model, autoregressive moving average (ARMA) model, and autoregressive integrated moving average (ARIMA) model.

These models are well suited for time-series predictions, but they lack a good description of the fractal nature of network traffic.

Manuscript received September 23, 2005; revised December 23, 2005; accepted June 14, 2006. Paper no. TII-05-09-0055.R1.

J. Kolbusz is with the Department of Distributed Systems, University of Computer Technology and Management, Rzeszów, Poland (e-mail: jkolbusz@wsiz. rzeszow.pl).

S. Paszczyński and B. M. Wilamowski are with the Department of Distributed Systems, University of Computer Technology and Management, Rzeszów, Poland (e-mail: spaszczynski@wsiz.rzeszow.pl).

Digital Object Identifier 10.1109/TII.2006.885191
Asynchronous transfer mode cell relay networks are mostly used as backbones for the interconnection of enterprise networks composed of several LANs, thus leading to self-similar behavior of network traffic [13]. It was demonstrated in [10] that LAN traffic is statistically self-similar. Several self-similar models already developed are detailed below.

Fractional Brownian traffic (FBT) [23] is derived from classical Brown movement where distributions of movement increments are given by a normal distribution $B(s, t)=B(s)-B(t)$ with $N\left(0,(t-s)^{2 H}\right)$, where $0.5 \leq H \leq 1$ is the Hurst parameter. The probability distribution for fractional Brownian traffic is given by

$$
f_{H}(x, t-s)=\frac{1}{\sqrt{2 \pi(t-s)^{2 H}}} \exp \left(-\frac{x^{2}}{2(t-s)^{2 H}}\right) .
$$

Fractional autoregressive integrated moving average (FARIMA) model [7], [12], [15] FARIMA $(0, d, 0)$ is a generalization of the ARIMA (p, d, q) model [3], [10], where p is the number of autoregressive terms, $\mathrm{d}$ is the number of nonseasonal differences, and $\mathrm{q}$ is the number of lagged forecast errors in the prediction equation

$$
\begin{aligned}
(1-B)^{d} & =\sum\left(\begin{array}{l}
d \\
k
\end{array}\right)(-1)^{k} B^{k} \\
\left(\begin{array}{l}
d \\
k
\end{array}\right) & =\frac{d !}{k !(d-k) !}=\frac{\Gamma(d+1)}{\Gamma(k+1) \Gamma(d-k+1)}
\end{aligned}
$$

where $\Gamma(\cdot)$ is the gamma function first introduced by Euler. The stream correlation is given by

$\rho_{t}(k)=\frac{\Gamma(1-d) \Gamma(k+d)}{\Gamma(d) \Gamma(k+1-d)} \sim \frac{\Gamma(1-d)}{\Gamma(d)} \frac{1}{|k|^{1-2 d}},|k| \rightarrow \infty$.

For FARIMA $(0, \mathrm{~d}, 0)$, the Hurst parameter is $0.5<H=$ $0.5+d<1$. This model was used for modeling a stream of compressed variable bit rate video [6].

A review of fractal models is given in [17]. In [2] and [26], it was shown that "on-off' models based on exponential or geometrical distributions do not fit well to traffic models. They do not exhibit fractal properties, and they are not self-similar. In the model presented is this paper, the Pareto distribution [20] was used, the resulting modeled properties of traffic on various layers are self-similar, and the model describes the fractal property of network traffic effectively.

This paper is an extended version of the presentation at INDIN'05 [9], and it is organized as follows. In Section II, a definition of self-similarity and estimation methods of self-similarity from measured data are given. Section III describes the network model with components significantly influencing network traffic. Experimental verifications are presented in Section IV. Section V presents a summary of this paper. 


\section{BACKGROUND}

With the introduction of the Internet, network traffic properties have changed significantly. Many modern traffic analysis lead to the conclusion that there is a stronger correlation in the stream of events than were earlier observed. At different time scales (milliseconds, seconds, hours), some correlations can be observed that are described by the term of self-similarity. Self-similarity is a property that is associated with fractals. It means that an object appears the same regardless of the scale at which it is viewed. A phenomenon that is self-similar looks the same or behaves the same when viewed at different degrees of magnification or different scales on a dimension. Self-similarity is defined as follows:

A stream of events

$$
\left\{t_{n}\right\}_{n=1}^{\infty}=t_{1}, t_{2}, \ldots, t_{n}, \ldots
$$

is self-similar [3], if the events' statistical properties are similar independently on the used time scale

$$
\left\{t_{n}^{(s)}\right\}_{n=1}^{\infty}=\left\{t_{1}^{(s)}, t_{2}^{(s)}, \ldots, t_{n}^{(s)}, \ldots\right\}
$$

where $\mathrm{s}=1,2,3, \ldots$ is the scale parameter. Streams of events are similar (not identical) if

$$
\left\{t_{n}^{(s)}\right\}_{n=1}^{\infty} \equiv\left\{t_{n}\right\}_{n=1}^{\infty} .
$$

Experimental verification of the long-term property of the stream of events is a variance decay of the averaged stream of events

$$
t^{(s)}=\frac{1}{s} \sum_{i=1}^{s} t_{i}=\frac{1}{s}\left(t_{1}+t_{2}+\cdots+t_{s}\right)
$$

which is slower than $1 / \mathrm{s}$; therefore

$$
\operatorname{Var}\left\{t^{(s)}\right\} \sim s^{-\beta}=\frac{1}{s^{\beta}}
$$

where $0<\beta<1$ is a coefficient describing function decay.

A stream of events (5) is exactly self-similar with parameter $0<\beta<1$, if the correlation function fulfills the following condition:

$$
\left\{\begin{array}{l}
\operatorname{Var}\left\{t^{(s)}\right\}=\frac{\sigma_{t}^{2}}{s^{\beta}} \\
\hat{\rho}_{t}^{(s)}(k) \equiv \hat{\rho}_{t}(k)
\end{array}\right.
$$

For every $\mathrm{s}, \mathrm{k}=1,2, \ldots$, where $\mathrm{s}$ is the time scale for subsequent $k$ time intervals between events.

Stream of events (5) is asymptotically self-similar with parameter $0<\beta<1$, if

$$
\left\{\begin{array}{l}
\operatorname{Var}\left\{t^{(s)}\right\}=\frac{\sigma_{t}^{2}}{s^{\beta}} \\
\hat{\rho}_{t}^{(s)}(k) \underset{s \rightarrow \infty}{\rightarrow} \hat{\rho}_{t}(k)
\end{array}\right.
$$

The measure of self-similarity is the Hurst parameter introduced by H. E. Hurst [8]. The Hurst parameter for self-similar processes is in the range of $0.5<H<1$. For two identical processes, $H=1$. Lower values of Hurst parameter indicate larger differences in processes, and for $H=0.5$, processes are not correlated (e.g., white noise). The Hurst parameter can be evaluated in several ways, primarily using R/S statistics [24]:

- using a rescaled adjusted range plot of $\mathrm{R} / \mathrm{S}$ as a function of time [1], [10], [15], [16];

- using a variance-time plot of R/S as a function of time [10], [15], [26];

- using a periodogram [15];

- using Wittle's estimator [15].

Simple "on-off" models are commonly used to describe the random nature of the network traffic [2], [26]. The "on-off" model successfully captures the second-order correlations of traffic, in particular their long range dependence (LRD). The "on" states are interlaced by the "off" states. The source transmits the packet in the "on" state, while the "off" state exists when there is no packet transmission. Therefore, the source works on the principle of a switch between the following active and inactive states.

Let $X(t), t>=0$ be the stationary process in which [11]

$$
X_{i}(t)=\left\{\begin{array}{ll}
1, & \text { for interval "on" } \\
0, & \text { for interval "off" }
\end{array} \quad i=1,2,3, \ldots, M .\right.
$$

The distribution of the time interval for the "on" states can be described by a Pareto distribution [20]. The distribution for the "on" states is given by

$$
\left\{\begin{array}{l}
F(t)=0 \\
1-F(t)=\left(\frac{d}{t}\right)^{\alpha} \quad t>d, \quad 1<d
\end{array}\right.
$$

where $d$ is the minimal time interval for an "on" state.

The distribution is heavy-tailed if large values of statistical variables occur with high probability and the following condition is met:

$$
\operatorname{Pr}\{X>x\}=1-F(x) \sim \frac{1}{x^{\alpha}}, \quad x \rightarrow \infty, \quad \alpha>0 .
$$

The average time interval for an "on" state for $1<\alpha<2$ is

$$
\bar{t}_{\mathrm{on}}=\frac{\alpha d}{\alpha-1}
$$

The time interval for an "off" state is described by a classical random distribution with a mean value $\bar{t}_{\text {off }}$. The probability that the stream is in the "on" state is given by:

$$
p=\frac{\bar{t}_{\mathrm{on}}}{\bar{t}_{\mathrm{on}}+\bar{t}_{\mathrm{off}}} .
$$

The average intensity of stream components is

$$
E\left\{X_{i}(t)\right\}=p, \quad i=1,2, \ldots, M
$$


while the resultant intensity is

$$
E\left\{\sum_{i=1}^{M} X_{i}(t)\right\}=M p .
$$

In the case when the function

$$
x(t)=\sum_{i=1}^{M} X_{i}(t)
$$

is asymptotically self-similar (11)

$$
\begin{aligned}
& \lim _{s \rightarrow \infty} \hat{\rho}_{t}^{(s)}(k)=\hat{\rho}_{t}(k) \\
& \quad=\frac{1}{2}\left[(k+1)^{3-\alpha}-2 k^{3-\alpha}+(k-1)^{3-\alpha}\right], k>0
\end{aligned}
$$

and

$$
\hat{\rho}_{t}(-k)=\hat{\rho}_{t}(k), \quad k<0
$$

then

$$
\lim _{|k| \rightarrow \infty} \hat{\rho}_{t}(k)=\frac{1}{|k|^{\alpha-1}} .
$$

Assuming that the correlation function decays exponentially

$$
\hat{\rho}_{t}(k) \sim \frac{1}{|k|^{\beta}}, \quad 0<\beta<1
$$

and

$$
H=1-\frac{\beta}{2}
$$

then

$$
H=\frac{3-\alpha}{2} H .
$$

The typical switching between 1 and 0 states ("on" and "off" states) is random. This randomness reflects user behavior at a computer as well as the applications employing the network services. The traffic "on-off" model is also connected with the client-server model. In the "on" state, the client sends a request to the server and the server is expecting a request. In the "off" state, the client waits for a response and the server is generating a response. Lengths of the "on" and "off" states are random, as well as the length of neighboring "on-off" intervals.

The superposition of such sources results in the LRD observed in network traffic. This was described in [25] where the "on-off" traffic models were derived as a superposition of a large number of "on-off" sources, with heavy-tailed "on" and/or "off" periods. Authors of [18] and [19] presented a new model called "alpha-beta on-off model," which is a composition of two different "on-off" models.

There is a strong tendency for adaptation of TCP/IP protocols for industrial environments [14]. This technology is used in industry dedicated network technologies due to both significant increase of TCP/IP throughput and its easy integration with enterprise resource planning (ERP) systems, which need an open environment [5]. In industrial environments, there are many real time processes, and these create additional constraints on the

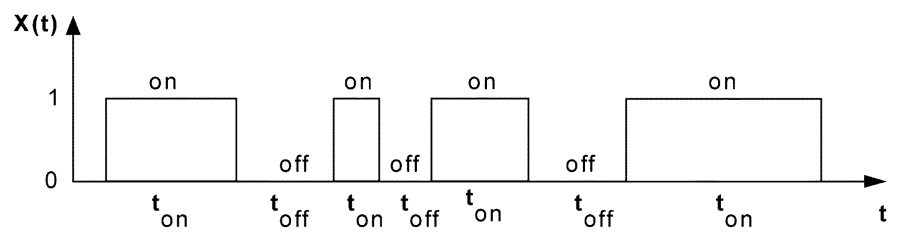

Fig. 1. Example of an "on-off” process.

network protocols. For the more optimal use of network protocols, a better understanding of the network traffic and its origin are required. New, better network models would be essential for easier adaptation of TCP/IP protocols in industry.

\section{Proposed TRafFic Model}

With access to remote multimedia information libraries (video and sound), www-page searching and increasing number of e-business applications, human behavior has a significant impact on network traffic. The usage of the network is mainly based on the analysis of the information resources and on their transfer. The whole process can be conveyed to a simple model of "on" (connecting) and "off" (listening) processes (see Fig. 1).

It is obvious that relatively intense network LAN traffic is generated when users start their work. At the beginning of the day, most of the internet traffic is related to various e-mail services. Later, other kinds of traffic like web browsing, file transferring, and remote computer operation become dominant. Another characteristic feature is the tendency to copy the day's schedule, which results frequently from a company internal rules and work time set up. In consequence of wide-scale marketing actions or the latest trend, it happens that in a very short period of time, the number of visitors on one server grows exponentially.

The traffic burst, for example, can be due to people's preferences caused by an advertisement. Consequently, human behavior is a very important aspect to understand the causality of the self-similar phenomenon originating in network traffic. Therefore, self-similarity in network traffic cannot be explained without deep analysis of the way individuals use the network. Self-similarity is not only conditioned by transmission protocols and computer systems, but it is also strictly connected with such disciplines like psychology and sociology.

In order to better describe network traffic, the model must take into account all essential factors that influence that traffic. To achieve a correct description of network traffic, several factors such as human behavior, the properties of the operating system, the process scheduling algorithm, and features of transmission protocols have to be analyzed. Most network applications use the TCP/IP protocol, and this has a significant impact on the shape of network traffic. The information between the user's application and the physical layer is sequentially converted by several processes such as coding, fragmentation, buffering, and adding of the header and tail. Thus, communication between processes has significant influence on the traffic. The proposed model, as shown in Fig. 2, is based on the ISO/OSI network model.

Individual components of the model represent network traffic generators with different frequency ranges for "on-off" processes. The proposed model was verified by measuring the 


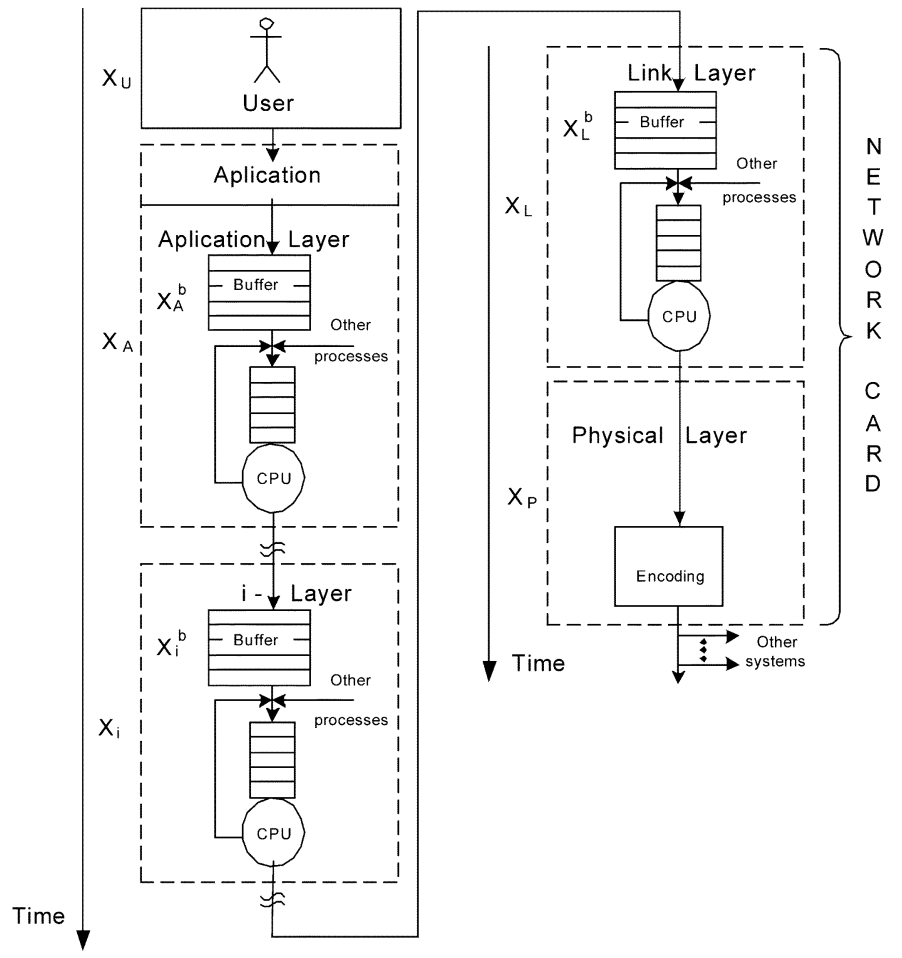

Fig. 2. Network model with components significantly influencing the network traffic, where $\mathrm{X}_{\mathrm{U}}, \mathrm{X}_{\mathrm{A}}, \mathrm{X}_{\mathrm{i}}, \mathrm{X}_{\mathrm{L}}, \mathrm{X}_{\mathrm{P}}$ are "on-off" processes depending on the user behavior, the application and application layer, intermediate layers, the link layer, and the physical layer, respectively.

Hurst parameter for network traffic for different time scale (s) changing from $10^{3} \mathrm{~s}$ to $10^{-3} \mathrm{~s}$.

Higher frequency analysis $(>10 \mathrm{~Hz})$ shows an influence of protocol algorithms, the operating system, and information buffering. For example, in a frequency of ready processes (i.e., processes in the run queue), context switches vary from about 10 to $100 \mathrm{~Hz}$. The influence of the particular components on the shape of corresponding function $\mathrm{X}(t)$ is presented in Fig. 3 .

In the model shown in Fig. 2, the components can be characterized by "on-off" functions, which are switched with different frequencies $(f)$ in the following domains:

$\mathrm{X}_{\mathrm{U}}$-human behavior with $f \leq 10^{-2} \mathrm{~Hz}$;

$\mathrm{X}_{\mathrm{A}}$ - the network processes embedded in a network application with $10^{-2} \leq f \leq 1 \mathrm{~Hz}$

$\mathrm{X}_{\mathrm{OS}}$ - the queuing of the processes to the CPU with $10 \leq$ $f \leq 10^{2} \mathrm{~Hz}$

$\mathrm{X}_{\mathrm{i}}$ - the $i$ th process realizing information conversion accordingly to network layer protocols with $10 \leq f \leq 10^{3}$ $\mathrm{Hz}$;

$\mathrm{X}_{\mathrm{L}}$-link layer with $f \geq 10^{3} \mathrm{~Hz}$;

$\mathrm{X}_{\mathrm{P}}-$ signal in the physical layer.

The electrical equivalent of the model is shown in Fig. 4.

Taking into account that in the physical layer the observed traffic is a superposition of the components mentioned above, the following formula for its description is proposed:

$$
X_{P}\left(t+\Delta t_{\mathrm{ps}}\right)=\prod_{i=1}^{n}\left[X_{i}\left(t+\Delta t_{p_{i}}\right)+X_{i}^{b}\left(t+\Delta t_{p_{i}}^{b}\right)\right]
$$

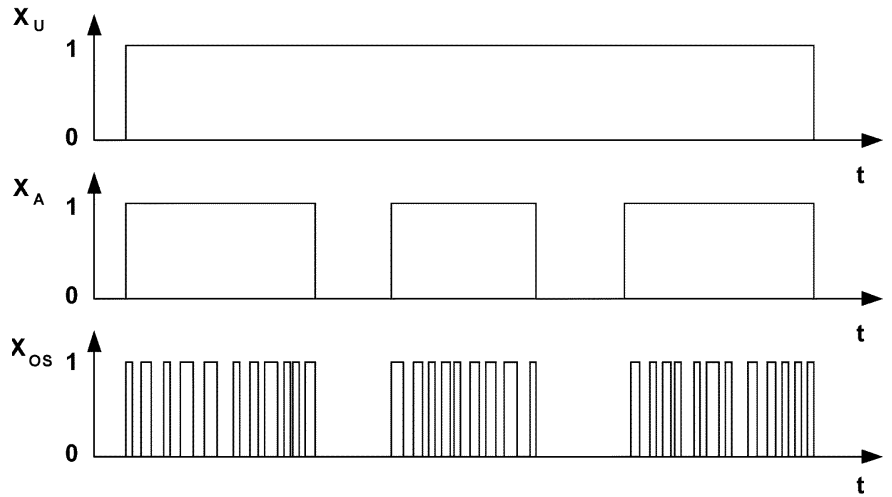

Fig. 3. Example of various "on-off" functions modeling network traffic generators: $\mathrm{X}_{\mathrm{U}}$-the user, $\mathrm{X}_{\mathrm{A}}$-applications and application layer, and $\mathrm{X}_{\mathrm{OS}}$-operating system, respectively.

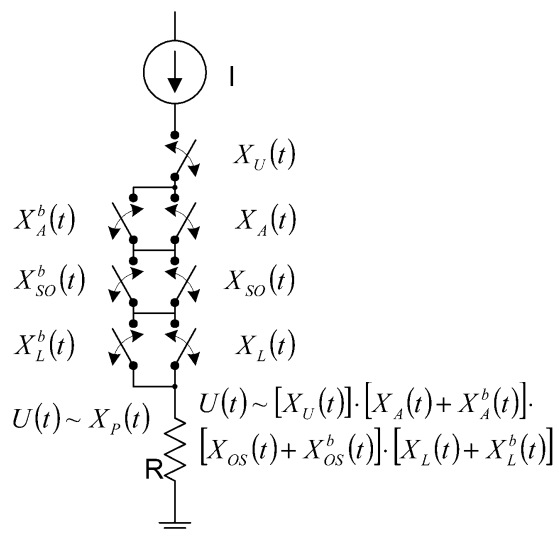

Fig. 4. Electrical equivalent of the model from Fig. 2.

where $X_{i}$ is the function describing behavior of $i$ th layer, $X_{i}^{b}$ is the function describing behavior of $i$ th buffer, $\Delta t_{p_{i}}$ is the time of signal propagation in the computer system, and $\Delta t_{p_{i}}^{b}$ is the time of signal propagation in the buffers.

The traffic in various communication layers of the "on-off" model is generated in the following way.

- Traffic in each layer is generated according to the "on-off" model for the given probability distribution, e.g., Pareto distribution, and system behavior (processes queuing and buffering).

- In the case of the first "on" period, the starting time for sublayers is randomly chosen in order to avoid the case that the first period starts at the beginning of the "on" period of the higher layer.

- The resultant traffic is a superposition of processes of all layers of the model.

- For the last layer for the "on" process, the size of the packet is generated using an adequate probability distribution such as Pareto, exponential, etc.

Assuming that the signal propagation time at every layer is much smaller than the period of the "on" state, one may write

$$
\Delta t_{\mathrm{pi}}, \Delta t_{\mathrm{pi}}^{b} \ll t_{\mathrm{ON}}, t_{\mathrm{OFF}}
$$


thus from (25), neglecting the propagation times $\Delta t_{p_{i}}$ and $\Delta t_{p_{i}}^{b}$

$$
X_{P}(t) \approx \prod_{i=1}^{n}\left[X_{i}(t)+X_{i}^{b}(t)\right] .
$$

Considering components of the model

$$
\begin{aligned}
& X_{P}(t) \approx\left[X_{U}(t)\right] \cdot\left[X_{A}(t)+X_{A}^{b}(t)\right] \\
& \cdot\left[X_{\mathrm{OS}}(t)+X_{\mathrm{OS}}^{b}(t)\right] \cdot\left[X_{L}(t)+X_{L}^{b}(t)\right]
\end{aligned}
$$

and assuming that maximum throughput is equal to $p_{\max }$, one can obtain throughput $I(t)$ from (29) as

$$
I(t) \approx X_{P}(t) \cdot p_{\max }
$$

where $p_{\max }$ is a property of the communication channel representing the maximum amount of information in bits, which can be transmitted per one second. This is a constant value of a given type of the network, for example $100 \mathrm{Mbs}, 1000 \mathrm{Mbs}$, etc.

An average throughput $\overline{I(t)}$ can be obtained from

$$
\overline{I(t)}=\frac{1}{t_{2}-t_{1}} \int_{t_{1}}^{t_{2}} I(t) d t
$$

where $\left[t_{1}, t_{2}\right]$ is the time interval of observation.

The effect of the operational system delay can be introduced by generation delays, which are dependant on the time required for information processing, setting queues, and buffering.

\section{EXPERIMENTAL VERIFICATION}

Experimental verification of the proposed model was done on the computer network of the University of Information Technology and Management in Rzeszow, Poland (see Fig. 5). This network has $550 \mathrm{PC}$ computers, two routers, and 30 servers. The collected data indicate that the level of traffic was similar during weekdays and during weekends. This can be explained by the specific operation of this university. Extended time data collection (over several months) was done to illustrate the self-similarity effect for long periods of operation. It is worth noticing that the traffic level was strongly influenced by the human factor since people are the major initiators of network traffic.

Statistical analysis of the network traffic during night hours (0:01 to 6:00 A.M.) shows that the Hurst parameter is in the range $0.51-0.58$, which indicates that the night time traffic is softly correlated and that self-similarity is low. This type of traffic is similar to white noise. During day hours (6:00 A.M. to 11:59 P.M.), the Hurst parameter is greater than 0.6 , which indicates strong self-similarity.

The following equipment was used for measuring the traffic in the LAN network:

Process snoop-network operating system UNIX Solaris 9, Link View Classic - the special card for the analysis of network traffic. The LinkView Classic Network Analyzer is a self-contained, software-only LAN analyzer that works with most third-party Ethernet or Token Ring Network adapter cards.

The typical shape of the traffic in the LAN is shown in Figs. 6-8.

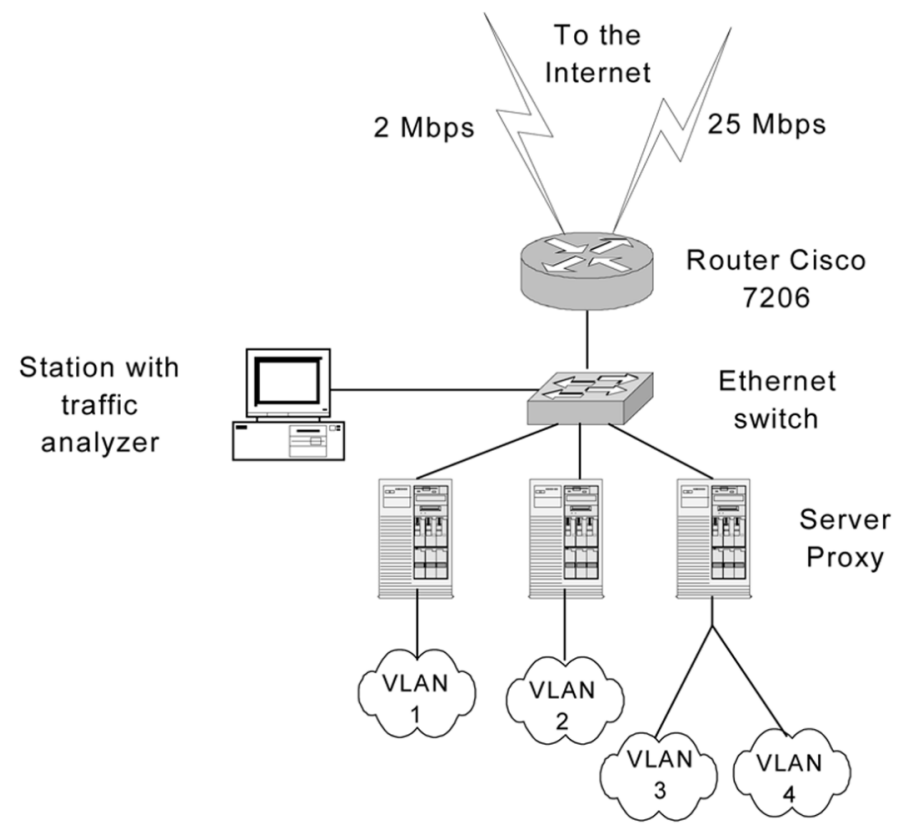

Fig. 5. LAN configuration of the University of Information Technology and Management in Rzeszow, Poland.

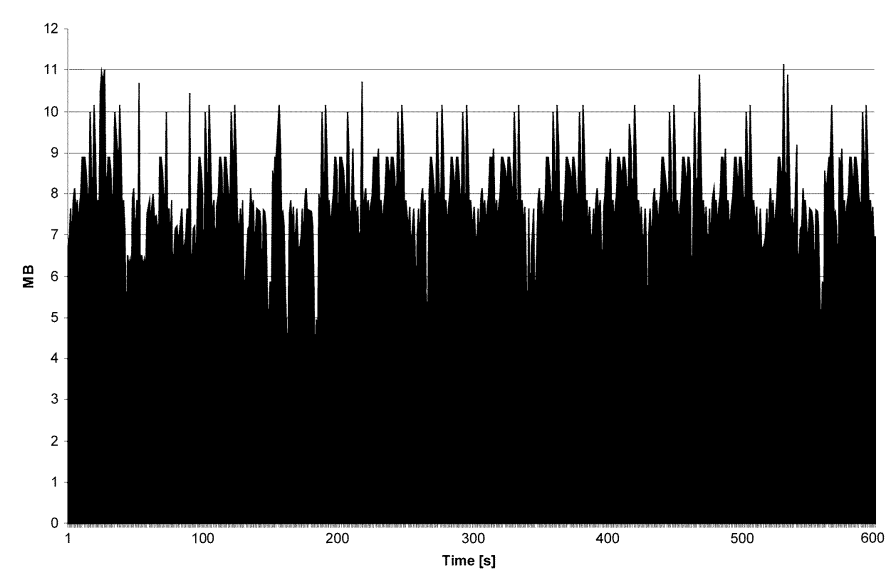

Fig. 6. Real traffic shape in the LAN.

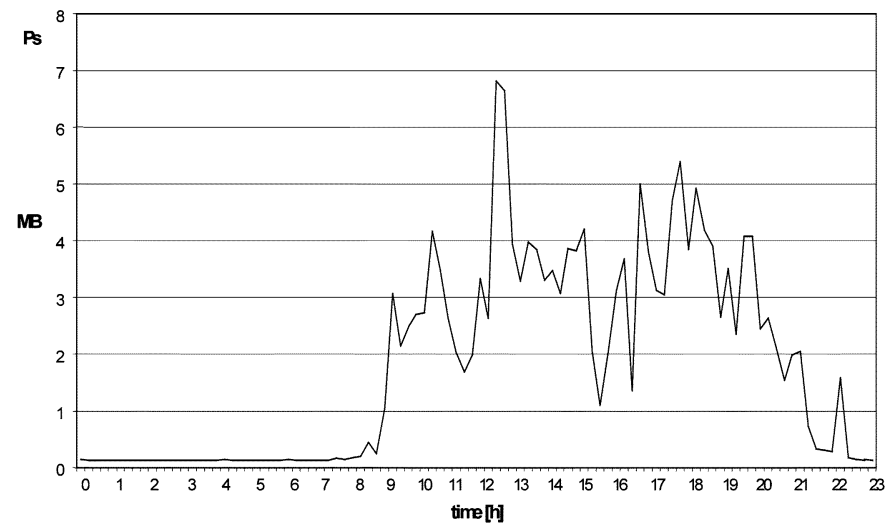

Fig. 7. Example of night and day traffic to a selected LAN server.

To check the influence of the processor load on the generation of traffic in the LAN, data were collected for sending a file (150 MB) using the FTP protocol, between two computers (Sun 


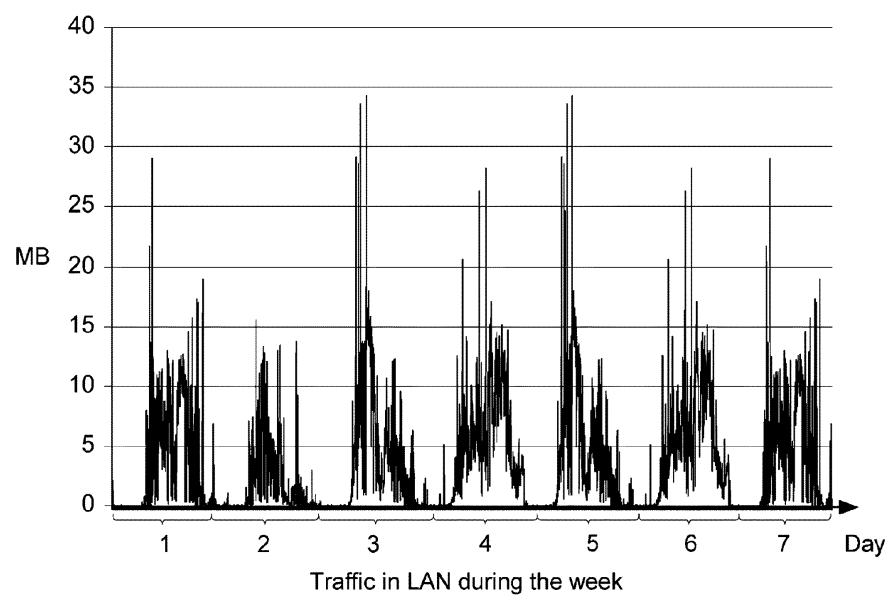

Fig. 8. Real traffic in the LAN during a week to the University of Information Technology and Management in Rzeszow, Poland. The measurements started Sunday at midnight (time gradation $\Delta t=60 \mathrm{~s}$ ).

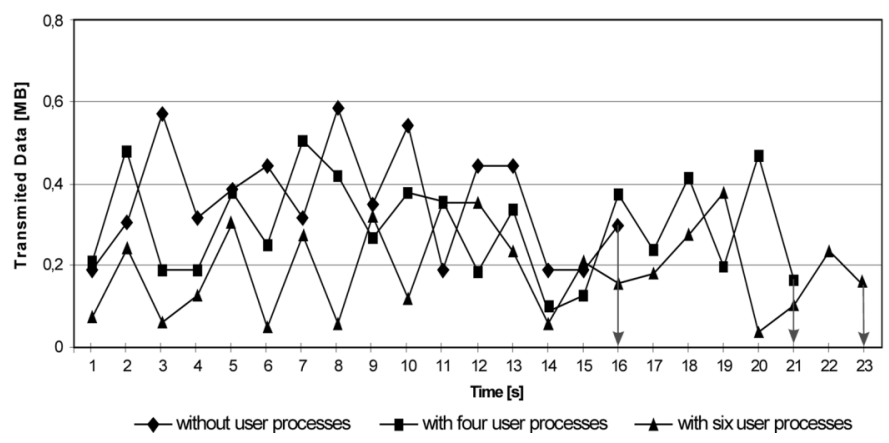

Fig. 9. Dependence of accumulated network traffic (gradation- $\Delta t=1 \mathrm{~s}$ ) in time; without user processes in queue to the CPU and with four and six user processes in queue to the CPU. (Color version available at http://ieeexplore. ieee.org.)

Blade 100, processor Spark $400 \mathrm{MHz}$, memory $256 \mathrm{MB}$, operating system: Unix Solaris 9). These measurements were done in two different conditions:

- station was loaded only by the system and the transmission processes station;

- additionally loaded with 4 and 6 user processes.

It was observed that with the increase of server load originated by user processes, the transmission time of information increases. At the same time, the level of traffic slightly decreases. The influence of the processor load on the traffic LAN is presented in Fig. 9.

To investigate the self-similarities of the traffic, the traffic was analyzed under different gradations $\Delta t$ conditions. The results are shown in Figs. 10 and 11.

The traffic of a computer network is a very complex self-similar phenomenon, dependent on many factors and consisting of components with frequencies in a wide range. The highest frequency of the traffic is generated by the link layer, while human action is a low-frequency component generator. The obtained results show that a major influence on traffic self-similarity is human behavior as a network traffic generator. This conclusion can be drawn from the high values of the Hurst parameter for low frequencies (see Table I). Because human behavior has a low-frequency character and the observed Hurst coefficient
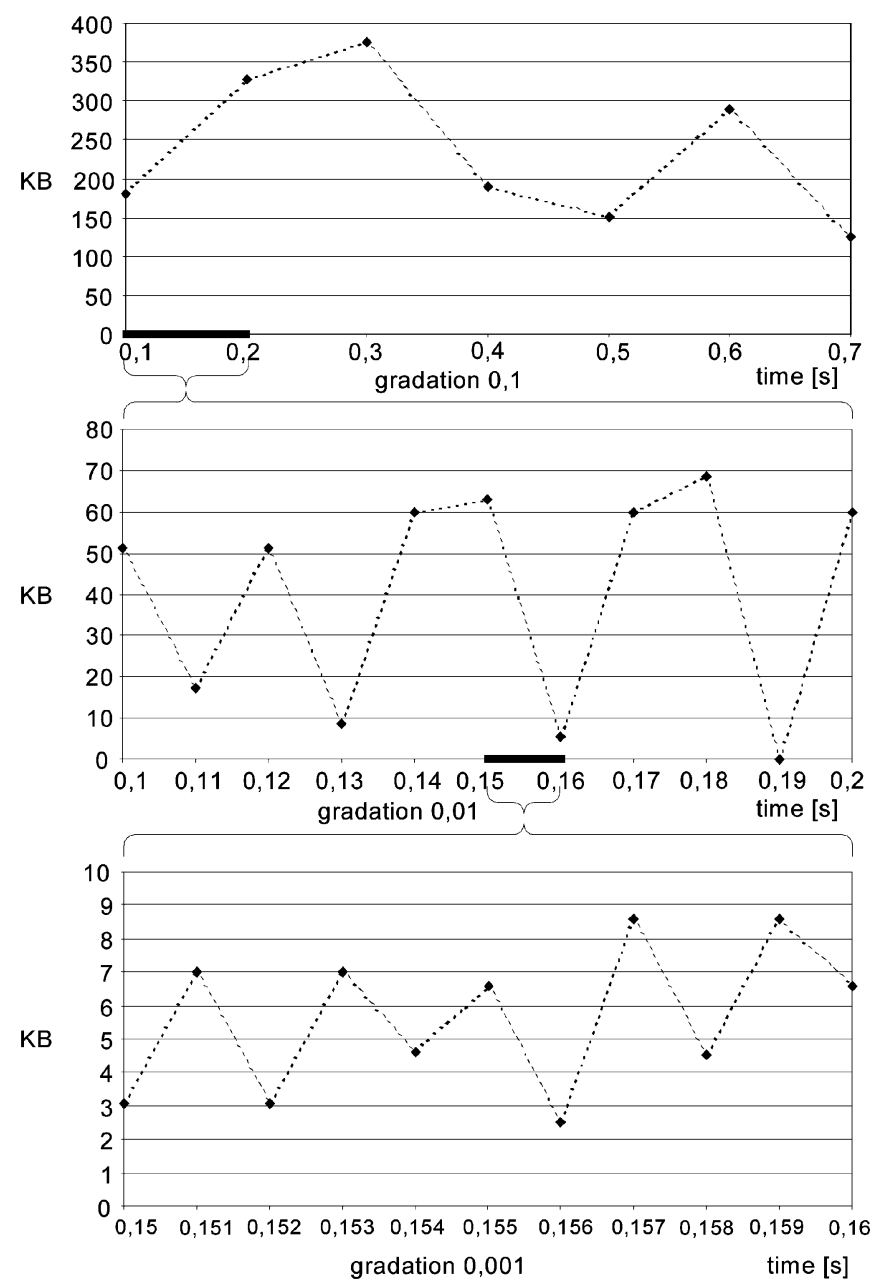

Fig. 10. Total transmitted data for different gradation of the time measurement and traffic accumulation.

for these frequencies is high, it confirms that human action increases self-similarity.

One may also notice that for the extended observation period (small frequencies), the Hurst parameter $H \Rightarrow 1$, and this means that the traffic is self-similar. In cases of short observation times (high frequencies), the value of the Hurst parameter $H \Rightarrow 0.5$, and this means that traffic not self-similar-signals are not correlated, and they have a white noise character.

The standard deviation for the experimental results is lower than 0.03 for both methods ( $\mathrm{R} / \mathrm{S}$ analysis and Variance Plot). Since both methods produce similar values of Hurst parameters $\mathrm{H}$, one can consider them trustworthy.

The Hurst parameter also can be used to detect protocol faults. For example, a disadvantage of the TCP protocol can be a faulty operation of the Nagle algorithm (RFC 896). This algorithm limits the number of small segments sent, especially in systems with large delays. A small segment is one that is smaller than the maximum segment size (MSS). The Nagle algorithm functions in the following manner. A single segment is sent and awaits an accept signal. If, while waiting for the ACK signal, new single small segments are generated, these segments are combined into one large segment. In the case that the combined segment grows to full size (MSS), it is sent immediately, as long as the receive 

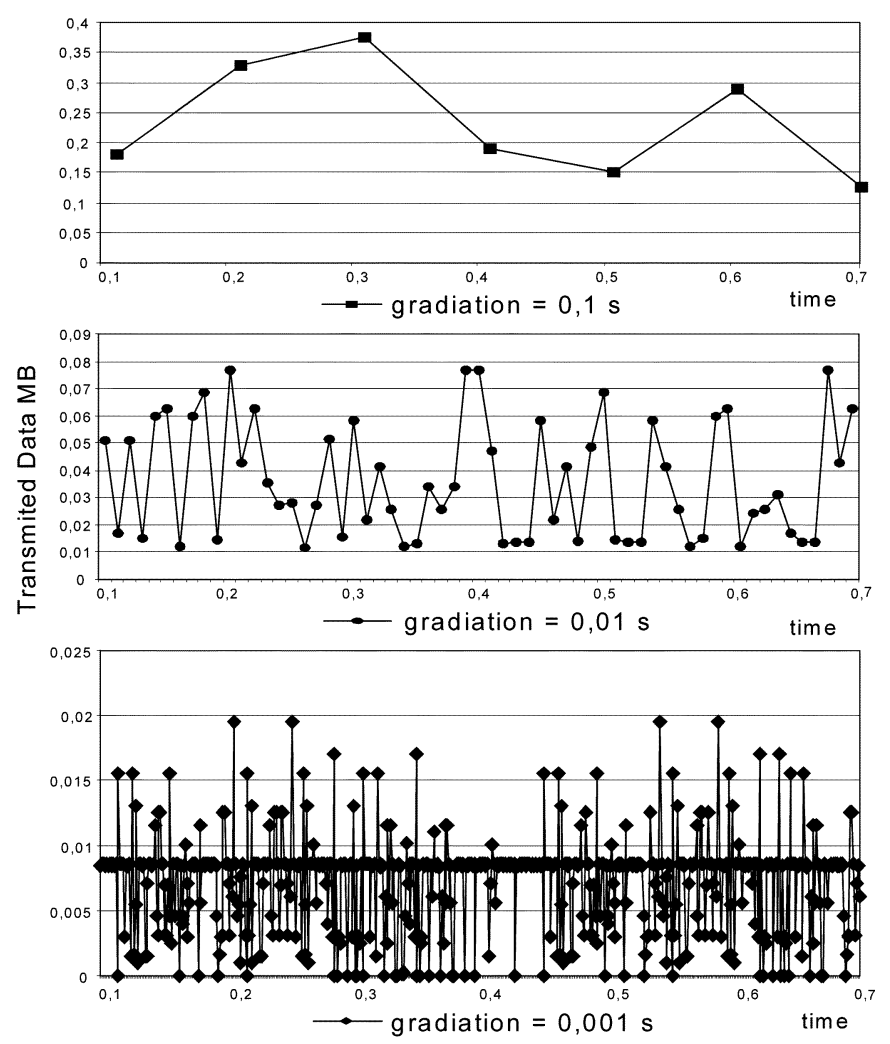

Fig. 11. Graph of transmitted data for different time of averaging.

TABLE I

HURST PARAMETER AS A FUNCTION OF FREQUENCY ESTIMATED By R/S AND VARIANCE PlOt ANALYSIS FOR THE RESUlTS OF THE TRAFFIC MEASUREMENTS IN LAN

\begin{tabular}{|l|l|l|}
\hline $\begin{array}{l}\text { Sampling frequency } \\
{[\mathrm{Hz}]}\end{array}$ & $\mathrm{R} / \mathrm{S}$ analysis & Variance Plot \\
\hline 0.001 & 0.91 & 0.89 \\
\hline 0.01 & 0.84 & 0.86 \\
\hline 0.1 & 0.79 & 0.78 \\
\hline 1 & 0.69 & 0.66 \\
\hline 10 & 0.64 & 0.63 \\
\hline 100 & 0.58 & 0.57 \\
\hline 1000 & 0.54 & 0.53 \\
\hline
\end{tabular}

window is large enough. The Nagle algorithm effectively decreases the number of packets sent, especially in the case of interactive applications such as Telnet, HTTP, etc. This is particularly important in cases of slow network connection.

\section{RECAPITULATION AND CONCLUSIONS}

Experimental results indicate that self-similarity processes with large Hurst parameters are primarily generated by human behavior. For example, self-similarity is observed in long processes (minutes and hours), while for short time intervals (milliseconds), processes have white noise characteristics. One may also notice a much stronger self-similarity effect during daytime, while during night when traffic is primarily generated by non-human, all processes are less correlated.

Our investigation verified that the proposed model qualitatively agrees with the observed traffic and includes the most important components-traffic generators. Further research, both on human behavior when using network applications as well as on transmission protocols running under an operating system, should give us the possibility to precisely model the $X(t)$ function for all considered model components. Based on this concept, a traffic model generator was developed. Currently, it may represent a single computer in the network. It is our intention to extend this generator for multiple computers and to develop a system of traffic simulation for an entire network.

Initial results indicate that optimization of buffer sizes and network processing parameters (transmission protocols) should improve traffic quality - decreasing both the fragmentation and time of transmission.

\section{REFERENCES}

[1] J. Beran, Statistics for Long-Memory Processes. New York: Chapman \& Hall, 1994.

[2] M. Crovella and A. Bestavros, "Self-similarity in world wide web traffic. Evidence and possible causes," IEEE/ACM Trans. Netw., vol. 5, no. 6, pp. 835-846, Dec. 1997.

[3] A. K. Erlang, "The theory of probabilities and telephone conversations," Nyt Tidsskrift fur Matematik, B., vol. 20, p. 33, 1909.

[4] V. S. Frost and B. Melamed, "Traffic modeling for telecommunications networks," IEEE Commun. Mag., vol. 32, no. 3, pp. 70-81, Mar. 1994

[5] Y. Genovese, B. A. Bond, B. Zrimsek, and N. Frey, "The transition to ERP II: Meeting the challenges," Gartner Res., 2001, R-14-0612.

[6] C. Huang, M. Deveksitiotis, I. Lambadaris, and A. R. Kaye, "Modeling and simulation of self-similar variable bit rate compressed video: A unified approach," in Proc. ACM SIGCOMM, Cambridge, MA, 1995, pp. $114-125$

[7] J. Ilow, "Forecasting network traffic using FARIMA models with heavy tailed innovations," in Proc. IEEE Int. Conf. Acoustics, Speech, Signal Processing, 2000, pp. 3814-3817.

[8] H. Kettani and J. A. Gubner, "Novel approach to the estimation of the hurst parameter in self-similar traffic," in Proc. IEEE Conf. Local Computer Networks, Nov. 2002, pp. 1-6.

[9] J. Kolbusz, S. Paszczyński, and M. Wilamowski, "Network traffic model for industrial environment," in Proc. IEEE Int. Conf. Industrial Informatics, Perth, Australia, Aug. 10-12, 2005, pp. 191-196.

[10] W. E. Leland, M. S. Taqqu, W. Willinger, I. Daniel, and V. Wilson, "On the self-similar nature of ethernet traffic (extended version)," IEEE/ACM Trans. Netw., vol. 2, no. 1, pp. 1-15, Feb. 1994.

[11] M. Likhanov, B. Tsybakow, and N. D. Georganas, "Analysis of an ATM buffer with self-similar (fractal) input traffic," in Proc. IEEE INFOCOM, Boston, MA, Apr. 1995, pp. 982-985.

[12] J. Liu, O. Yang, Y. Shu, F. Xue, and L. Zhang, "Traffic modeling based on FARIMA models," in Proc. CCECE, Edmonton, AB, Canada, May 1999, pp. 162-167.

[13] V. Paxson and S. Floyd, "Wide area traffic: The failure of poisson modeling," IEEE/ACM Trans. Netw., vol. 3, no. 3, pp. 226-244, Jun. 1995.

[14] N. Pereira, F. Pacheco, L. M. Pinho, A. Prayati, E. Nikoloutsos, A. Kalogeras, E. Hintze, H. Adamczyk, and L. Rauchhaupt, "Integration of TCP/IP and PROFIBUS protocols," in Proc. 4th IEEE Int. Workshop Factory Communication Systems, Sweden, 2002.

[15] A. Popescu, "Traffic self-similarity," in Proc. IEEE Int. Conf. Telecommunications, Bucharest, Romania, Jun. 2001, pp. 20-24.

[16] J. Roberts, U. Mocci, and J. Virtamo, "Broadband network traffic," in Final Report of Action COST 242. Berlin, Germany: Springer-Verlag, 1996.

[17] B. Ryu and S. Lowen, "Fractal traffic models for internet simulation," in Proc. 5th IEEE Symp. Computers Communications, Jul. 2000, pp. 200-206.

[18] S. Sarvotham, R. Riedi, and R. Baraniuk, "Connection-level analysis and modeling of network traffic," in Proc. IEEE/ACM Internet Measurement Workshop, 2001, pp. 1-5.

[19] S. Sarvotham, R. H. Riedi, and R. G. Baraniuk, "Network and user driven on-off source model for network traffic," Comput. Netw. J., Special Issue on "Long-range Dependent Traffic", pp. 335-350, Oct. 2005.

[20] W. Stalings, High-Speed Networks. TCP/IP and ATM Design Principles. Upper Saddle River, NJ: Prentice-Hall, 1998.

[21] M. S. Taqqu, W. Willinger, and R. Sherman, "Proof of a fundamental result in self-similar traffic modeling," Comput. Commun. Rev., vol. 27, no. 2, pp. 5-23, 1997. 
[22] B. Tsybakov and N. D. Georganas, "On self-similar traffic in ATM queues: Definitions, overflow probability bound, and cell delay distribution," IEEE/ACM Trans. Netw., vol. 5, no. 3, pp. 397-409, Jun. 1997.

[23] A. Vidács and J. T. Virtamo, "Parameter estimation of geometrically sampled fractional brownian traffic," in Proc. IEEE INFOCOM, TelAviv, Israel, Mar. 2000, pp. 26-30.

[24] J. R. Wallis, "Robustness of the rescaled range R/S in the measurement of noncyclic long-run statistical dependence," Water Resour. Res., vol. 5, pp. 967-988, 1969.

[25] W. Willinger, V. Paxson, R. Riedi, and M. Taqqu, "Long range dependence: Theory and applications, chapter," in Long Range Dependence and Data Network Traffic. New York: Wiley, 2002.

[26] W. Willinger, M. Taqqu, R. Sherman, and D. Wilson, "Self similarity through high-variability: Statistical analysis of Ethernet LAN traffic at the source level," IEEE/ACM Trans. Netw. (Extended Version), vol. 5, no. 1, pp. 71-86, Feb. 1997.

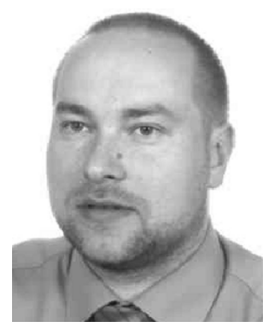

Janusz Kolbusz received the M.Sc. degree from the Faculty of Electrical and Computer Engineering at the University of Technology, Rzeszow, Poland, in 1998.

He worked as an Assistant in the Department of Distributed Systems, University of Computer Science and Management, Rzeszow. His teaching and research interests are in the operating systems, computer networks, and architectural systems development. He currently works on LAN traffic modeling.

Since 2000, Mr. Kolbusz has been a member of PTI, the Polish Information Processing Society.

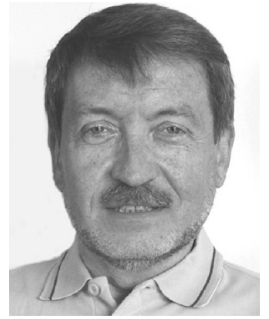

Stanisław Paszczyński (SM'05) received the M.S., Ph.D., and D.Sc. degrees in electronics from Warsaw University of Technology, Warsaw, Poland, in 1972, 1979 , and 1991, respectively.

In 1985, he was with the Microelectronics Institute of Catholic University, Leuven, Belgium. From 1989 to 1991 , he was a Visiting Assistant Professor with the Science Department, University of Texas, San Antonio. From 1992 to 1999, he was a Professor in the Electrical Department of Rzeszow University of Technology, Rzeszow, Poland. He is currently an Associate Professor at the University of Computer Science and Management, Rzeszow, where he works on network traffic analysis and modeling as well as on agent technology use in the network throughput increase.

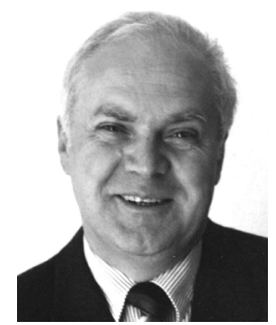

Bogdan M. Wilamowski (M'82-SM'83-F'00) received the M.S. degree in computer engineering in 1966, the Ph.D. degree in neural computing in 1970, and the D.Sc. degree in integrated circuit design in 1977.

He received the title of Full Professor from the President of Poland in 1987. He was the Director of the Institute of Electronics (1979 to 1981) and the Chair of the Solid State Electronics Department (1987 to 1989) at the Technical University of Gdansk, Gdansk, Poland. He was a Professor at the University of Wyoming, Laramie, from 1989 to 2000. From 2000 to 2003, he was an Associate Director of the Microelectronics Research and Telecommunication Institute at University of Idaho, Moscow, and he was a Professor in the Electrical and Computer Engineering Department and in the Computer Science Department at the University of Idaho. Currently, he is the Director of the Alabama Microelectronics Science and Technology Center (AMSTC) and a Professor of the Electrical and Computer Engineering Department at Auburn University, Auburn, AL. He was with the Communication Institute at Tohoku University, Sendai, Japan, from 1968 to 1970 , and he spent one year at the Semiconductor Research Institute, Sendai, as a JSPS Fellow (1975 to 1976). He was a Visiting Scholar at Auburn University (1981 to 1982 and 1995 to 1996) and a Visiting Professor at the University of Arizona, Tucson (1982 to 1984).

Dr. Wilamowski was the President of the IEEE Industrial Electronics Society from 2004 to 2005 . He served as an Associate Editor of IEEE TRANSACTIONS ON NEURAL NETWORKS, IEEE TRANSACTIONS ON EDUCATION, IEEE TRANSACTIONS ON INDUSTRIAL ELECTRONICS, Journal of Intelligent and Fuzzy Systems, Journal of Computing, and International Journal of Circuit Systems. Currently, he is Editor-in-Chief Elect of IEEE TRANSACTIONS ON INDUSTRIAL ELECTRONICS. 